\title{
DISSIMILARITY CHARACTERISTIC THEOREMS FOR GRAPHS
}

\author{
FRANK HARARY ${ }^{1}$ AND ROBERT Z. NORMAN
}

A simple but general dissimilarity characteristic theorem was used in [1] to derive generating functions for counting various classes of graphs. We propose to use this result in a slightly generalized form, stated below as "Theorem," to give new and short derivations of Otter's dissimilarity theorem [7], its generalization to cacti [3] (formerly called Husimi trees), and an elementary theorem for graphs [2].

In general we follow standard terminology on graphs (König [6]); our graphs are finite, nonempty, and may have isolated points. A block of a graph ("Glied" in [6]) is a maximal connected subgraph containing no cut points of itself. For a given subgroup $\mathfrak{A}$ of the automorphism group of the graph, we say that two points are similar if a permutation of $\mathfrak{A}$ sends one point into the other. By "the number of dissimilar points" of a graph we mean the number of equivalence classes of similar points in it; analogously for lines, blocks, etc.

TheOREM. Let $G$ be a connected graph with $n$ similarity classes of blocks (with respect to a group $\mathfrak{R}$ ). Let $p$ be the number of dissimilar points of $G$ and $p_{k}$ be the number of dissimilar points in blocks of the kth similarity class. Then the following equation holds:

$$
p-1=\sum_{k=1}^{n}\left(p_{k}-1\right) \text {. }
$$

Proof. Exactly as in [1], but using similarity classes of points and blocks with respect to $\mathfrak{A}$.

We now specialize (1) to obtain the dissimilarity characteristic equation for trees. An exceptional line of a graph is one whose endpoints are similar.

Corollary 1. In any tree, let $p, q$, and $q^{e}$ be the numbers of dissimilar points, lines, and exceptional lines respectively; then

$$
p-\left(q-q^{e}\right)=1 .
$$

Received by the editors May 27, 1959.

${ }^{1}$ Work supported by a grant from the Office of Naval Research while on leave from the University of Michigan, and by the U.S. Atomic Energy Commission during the summer of 1959 while at the Los Alamos Scientific Laboratory. 
Proof. For the tree of one point, (2) is trivial. In any other tree, every block is a line, and at most one line is exceptional. Thus for each ordinary (nonexceptional) line constituting the $k$ th block of the tree, $p_{k}-1=1$, while for an exceptional line, $p_{k}-1=0$. Therefore, on summing over all $n$ dissimilar lines of a tree, we obtain

$$
\sum_{k=1}^{n}\left(p_{k}-1\right)=q-q^{e} .
$$

But on applying (1) the left side becomes $p-1$, proving the corollary.

A cactus is a connected graph in which no line lies in more than one cycle. ${ }^{2}$ Thus the blocks of a cactus (of more than one point) are lines or cycles. The dissimilarity characteristic equation for cacti is another special case of the theorem. To state this equation we require additional concepts. Let $Z$ be a cycle of a cactus $K$. Then $Z$ is pointsymmetric if there is an automorphism of $K$ in which exactly two points and no lines of $Z$ are fixed; similarly $Z$ is line-symmetric if there is an automorphism of $K$ which leaves exactly two lines and no points of $Z$ fixed. An axis of symmetry of $K$ is an exceptional line not in any cycle of $K$.

Corollary 2. Let $K$ be a cactus and let $p$ and $q$ be as in Corollary 1. Let $q^{a}$ be the number of axes of symmetry of $K$. Let $c$ be the number of dissimilar cycles of $K$ and let $c^{p}$ and $c^{q}$ be the number of dissimilar point-symmetric and line-symmetric cycles of $K$. Then the following equation holds:

$$
p-\left(q-q^{a}\right)+\left(c-c^{p}+c^{q}\right)=1 .
$$

Proof. Let us denote the number of dissimilar points, lines, and axes of symmetry of $K$ in the $k$ th block by $p_{k}, q_{k}$, and $q_{k}^{a}$ respectively. Also, let $c_{k}, c_{k}^{p}$, and $c_{\boldsymbol{k}}^{q}$ be the number of dissimilar cycles of the indicated kind in this block. We claim that (3) holds for this block:

$$
\left(p_{k}-1\right)-\left(q_{k}-\stackrel{a}{q_{k}}\right)+\left(c_{k}-\stackrel{p}{c_{k}}+\underset{c_{k}^{q}}{q}\right)=0 .
$$

This equation is easily verified separately for cycles that are pointsymmetric, line-symmetric, and neither. The proof can be found in [3]. On summing this equation over all dissimilar blocks of $K$ and applying equation (1) we obtain

$$
(p-1)-\left(q-q^{a}\right)+\left(c-c^{p}+c^{q}\right)=0,
$$

2 The term "cycle" in graph theory (cf. König [6]) stands for a specialization of the use of this term in combinatorial topology where "elementary cycle" indicates the same concept. 
proving the corollary.

The third corollary differs from the first two in that it does not refer to similarity classes.

CoROllary 3. In any connected graph $G$, let $N$ and $p$ be the number of blocks and points, and $n_{i}$ the number of components of the graph obtained on removing the ith point of $G$. Then

$$
N-\sum_{1}^{p} n_{i}+p=1 .
$$

Proof. To apply the theorem here, we define similarity with respect to the automorphism subgroup consisting only of the identity permutation. Then no two distinct points of $G$ are similar. With this stipulation, $p$ is also the number of dissimilar points of $G$.

Obviously

$$
\sum_{1}^{N}\left(p_{k}-1\right)=\sum_{1}^{N} p_{k}-N
$$

Now by (1) the left side is $p-1$. Each point $a_{i}$ of $G$ is in $n_{i}$ distinct blocks of $G$, one for each component formed by removing $a_{i}$ from $G$. Thus $\sum p_{k}=\sum n_{i}$ since each sum counts every point as many times as the number of blocks in which it lies. This proves Corollary 3.

It might seem that (1) could be applied efficiently in the proof of the dissimilarity characteristic equation for graphs [4]. However, applying (1) merely serves to reduce this equation to the case of graphs consisting of a single block; the proof in this case is just as difficult as for graphs in general.

\section{REFERENCES}

1. G. W. Ford, R. Z. Norman and G. E. Uhlenbeck, Combinatorial problems in the theory of graphs, II, Proc. Nat. Acad. Sci. U.S.A. vol. 42 (1956) pp. 203-208.

2. F. Harary, An elementary theorem on graphs, Amer. Math. Monthly vol. 66 (1959) pp. 405-407.

3. F. Harary and R. Z. Norman, The dissimilarity characteristic of Husimi trees, Ann. of Math. vol. 58 (1953) pp. 134-141.

4. - The dissimilarity characteristic of linear graphs, Proc. Amer. Math. Soc. vol. 5 (1954) pp. 131-135.

5. F. Harary and G. E. Uhlenbeck, On the number of Husimi trees, I, Proc. Nat. Acad. Sci. U.S.A. vol. 39 (1953) pp. 315-322.

6. D. König, Theorie der endlichen und unendlichen Graphen, Leipzig, 1936; reprinted, New York, 1950.

7. R. Otter, The number of trees, Ann. of Math. vol. 49 (1948) pp. 583-599.

Princeton University, InSTITUTE FOR AdVANCEd STUdy AND

Dartmouth College 